\title{
Un modelo conceptual para la realización del Trabajo Fin de Grado apoyado en el uso de las TICs
}

\section{A model for the realization of the Final Year Project Work supported in the use of the ITCs}

\author{
Úrsula Faura-Martínez \\ Universidad de Murcia. Murcia, España \\ faura@um.es \\ Pedro Juan Martín-Castejón \\ Universidad de Murcia. Murcia, España \\ pjmartin@um.es \\ Matilde Lafuente-Lechuga \\ Universidad de Murcia. Murcia, España \\ mati@um.es
}

\begin{abstract}
RESUMEN
En el actual escenario del ámbito universitario las herramientas de la tecnología y la comunicación (TIC) están siendo empleadas para favorecer nuevos enfoques metodológicos con un matiz claramente colaborativo proporcionando una importante proyección en los procesos de innovación educativa. Este trabajo describe una experiencia desarrollada en el ámbito universitario, cuyo objetivo es crear un modelo conceptual que refleje la influencia del trabajo colaborativo generado a través de las tutorías grupales y el uso de las TICs en el rendimiento académico en la elaboración del Trabajo Fin de Grado (TFG). Participaron estudiantes de los Grados en Administración y Dirección de Empresas y Marketing de la Universidad de Murcia durante tres cursos académicos. El 100\% de los estudiantes que siguieron esta metodología superaron con éxito la evaluación del TFG. El $40 \%$ obtuvo una nota superior a 9 , el $23,3 \%$ entre 8 y 9 y un $20 \%$ superó la evaluación con matrícula de honor. Se concluye que esta forma de desarrollar el proyecto basada en las tutorías grupales, el trabajo colaborativo y el uso de TIC favorece la elaboración del TFG, obteniendo unos resultados excelentes, produciéndose menos diferencias en las notas obtenidas y no hallándose ninguna influencia del sexo en dichos resultados.

Palabras clave: Aprendizaje colaborativo, TIC, innovación educativa, Trabajo Fin de Grado, coordinación interdisciplinar.
\end{abstract}

\begin{abstract}
:
In the current scenario of the university environment the tools of technology and communication (ICT) are being used to favor new methodological approaches with a clearly collaborative material providing an important projection in the processes of educational innovation. The present work describes an experience developed in the university level, which aims to create a conceptual model that can reflect the influence of collaborative work generated through group tutorials and the use of ICT in academic performance in the elaboration of the Final Year Project Work (for short, TFG). Graduates participated from Business Administration and Marketing of the University of Murcia during three academic courses. $100 \%$ of the students who followed this methodology successfully passed the evaluation of the TFG. $40 \%$ scored higher than $9,23.3 \%$ between 8 and 9 , and $20 \%$ passed the assessment with honors. It is possible to conclude that this way of developing the project based on group tutorials, collaborative work and the use of ICT favors the elaboration of the TFG, obtaining excellent results, producing less differences in the grades obtained between the students and not finding any influence sex in the results. Keywords: Collaborative learning, ICT, educational innovation, Final Year Project Work, interdisciplinary coordination.
\end{abstract}




\section{Introducción}

Para la implantación del EEES es necesaria una nueva reconceptualización de la formación académica superior, basada en el aprendizaje del alumno y en el diseño de herramientas metodológicas que favorezcan la adquisición de competencias. El futuro graduado no solo tiene que adquirir conocimientos, sino también habilidades que le preparen para su profesión. Por lo tanto, se debe formar al estudiante bajo un enfoque multidisciplinar para que aprenda a encontrar las sinergias entre conocimientos, habilidades y aptitudes adquiridas (Bonilla et al., 2012). Por ello es tan importante el aprendizaje interdisciplinar, ya que permite lograr un conocimiento más integral que el que se obtiene con el estudio de una asignatura específica, pues implica la interacción entre dos o más disciplinas, entre las que debe existir una eficiente intercomunicación (Puigcerver et al., 2013), donde las Tecnologías de la Información y la Comunicación (TICs) juegan un papel sumamente importante (Lara et al., 2014).

Para ello la coordinación y el trabajo en equipo del profesorado son elementos claves, ya que uno de los hábitos del profesorado universitario es su excesiva individualidad y especialización, que no le afectaba en el desarrollo de la clase magistral, pero si en el trabajo por competencias (Olaskoaga-Larrauri et al., 2015). En esta línea, el modelo conceptual que proponemos en el presente artículo recoge una experiencia de innovación educativa a través de la coordinación interdisciplinar de la asignatura Trabajo Fin de Grado (TFG), impartida en cuarto curso del Grado en Administración y Dirección de Empresas (ADE) y del Grado en Marketing en la Universidad de Murcia (Bote et al., 2013). Para el desarrollo de dicha experiencia se elige la estratégica metodológica asociada a la creación de un proyecto empresarial. Esta metodología nos permite formar un grupo de trabajo con todos los estudiantes de ADE y Marketing que eligen esta línea, que tienen que realizar un proyecto empresarial original, enfrentándose a una tarea real de creación de un proyecto.

Las implicaciones de nuestro estudio son dobles. Por un lado, aportamos una innovación metodológica en el ámbito universitario que permite a los estudiantes desarrollar competencias clave para su futuro profesional. Por otro lado, se fomentan los procesos de aprendizaje activo y de trabajo colaborativo entre el alumnado (Rullán et al., 2010). Además, ponemos de manifiesto la importancia de disponer de competencias vinculadas al uso de las TICs (Ricoy y Fernández, 2013).

En el presente trabajo hace referencia en primer lugar a la relevancia que tiene la interdisciplinaridad y el uso de las TICs en la realización del TFG, para ello hemos creado un modelo conceptual de relaciones entre las variables estudiadas.

\subsection{La tutoría grupal y el uso de las TICs}

El tutor es el que orienta, asesora y acompaña al estudiante durante la realización de su TFG (Rekalde, 2011), estimulando en él la capacidad de hacerse responsable de su propio aprendizaje y de su formación (García-Nieto, 2008). Por lo tanto, una de las principales funciones del tutor es facilitar la formación integral del estudiante. Para ello 
debe desarrollar estrategias que contribuyan a facilitar la adaptación del estudiante al ambiente de trabajo en su TFG, mejorar sus habilidades e incrementar su desempeño (Rodríguez y Llanes, 2013). Para que la relación entre tutor- alumno pueda darse de manera efectiva, es importante que exista comunicación efectiva entre ambos. En este sentido, el acompañamiento tutorial (Arbizu et al., 2005) es el resultado de un sistema previo de diseño y planificación didáctica, y su desarrollo supone también procesos de preparación de reuniones, actividades y formas de contacto que faciliten la puesta en circulación de los procesos y resultados del aprendizaje potenciado y activado el trabajo autónomo por parte del estudiante (Obaya y Vargas, 2014). La acción tutorial toma especial interés en el TFG (Arteaga et al., 2013), donde la labor fundamental del tutor es el diseño y la planificación de actividades significativas de aprendizaje que sean producto de la interacción con el estudiante a partir del uso y apropiación de recursos educativos enfocados a la realización de una tarea concreta (Castaño, 2011).

El proceso de acción tutorial puede llevarse a cabo de forma individual (el profesor con el alumno uno a uno) o hacerse de forma grupal (el profesor con grupos de alumnos que trabajan en torno a un tema común). Según Wisker (2012), las tutorías grupales son mejores ya que sirven para reforzar la planificación y el compromiso con el trabajo a realizar por parte del estudiante. La reflexión de grupo parece más eficaz, para la construcción de conocimiento compartido, que la individual, ya que las conclusiones tienen más puntos de vista.

En este contexto, lo que marca un antes y un después en la labor tutorial son las TICs, ya que posibilitan la relación entre los tutores con el alumnado y entre los propios tutores (Mishra et al., 2014). Estas herramientas otorgan una nueva dimensión por cuanto confieren la posibilidad de adaptarse a las necesidades que se presentan en cada momento. Por ello, las TICs han permitido la creación de nuevos entornos comunicativos que abren la posibilidad de desarrollar nuevas experiencias formativas y educativas (Pantoja y Zwierewicz, 2008).

\subsection{La influencia de las TICs en el trabajo colaborativo}

La división de las TICs en herramientas síncronas y asíncronas es la que garantiza la eficiencia comunicativa en todo momento, ya que sitúa la comunicación en un momento real o, por el contrario, permite a los interlocutores hacer intercambios en momentos temporales diferentes (Pantoja y Villanueva, 2010). Ambas características son muy útiles para el tutor, pues confieren a cada mensaje una mayor o menor urgencia, en función de las necesidades. Por lo tanto, las TICs facilitan la comunicación entre el tutor y los alumnos (Pedraza et al., 2013), pues favorece e incrementa los flujos de información y la colaboración entre ellos. De este modo, por ejemplo, cualquier alumno puede plantear una duda o realizar una consulta a su tutor desde cualquier lugar y en cualquier momento (Artola, 2012). De la misma forma, las TICs mejoran la comunicación entre alumnos, favoreciendo el aprendizaje cooperativo al facilitar la organización de actividades grupales (Ricoy y Fernández, 2013). Asimismo, cualquier tutor puede ponerse en contacto con otro tutor y planificar experiencias educativas de colaboración entre el alumnado. El trabajo colaborativo entre los tutores se verá 
fortalecido enormemente mediante el uso de las TICs, porque será necesario repartir las tareas y estar de acuerdo en las acciones que se deben seguir (Garzozi y Lucas, 2014). Al mismo tiempo, podrá existir un trabajo compartido con otros tutores y podrán intercambiar con ellos experiencias. Las TICs desempeñan un papel importante en este campo ya que pueden contribuir de manera significativa a profundos cambios en la Educación Superior (Barquilla, 2016).

\subsection{Comunidad de aprendizaje, trabajo colaborativo y grupo de Whatsapp}

Los entornos denominados como "comunidades de aprendizaje" son objeto de interesantes análisis (Onrubia, 2004). Específicamente en este estudio nos propusimos comprender más acerca de las posibilidades de desarrollo de una comunidad de aprendizaje para la realización del TFG. En su trabajo Coll (2001) entiende las comunidades de aprendizaje como un grupo de personas con diferentes niveles de experiencia y conocimiento, que aprenden mediante su implicación y participación en actividades, en función de la colaboración que establecen entre sí, en la construcción del conocimiento colectivo que desarrollan y de los diversos tipos de ayudas que se facilitan mutuamente. Las aulas como comunidades de aprendizaje permiten generar espacios de integración y un clima agradable entre compañeros y tutores (Wisker, 2012). Por lo tanto, una comunidad de aprendizaje supone una construcción del conocimiento que implica tanto procesos individuales como grupales, teniendo como principal objetivo mejorar el aprendizaje individual, y el aprendizaje en grupo, para lograr los objetivos propuestos (Mishra et al., 2014). Las cuatro condiciones necesarias para que se dé este trabajo colaborativo (Cenich y Santos, 2005) en una comunidad de aprendizaje son las siguientes:

1. En primer lugar, debe existir un objetivo común que suponga aprender algo nuevo como grupo.

2. En segundo lugar, debe haber responsabilidad individual. El éxito del trabajo en grupo reside en que todos los miembros del grupo se impliquen y aprendan en función de sus particularidades.

3. El esfuerzo de todos los miembros del grupo es valioso y necesario para la consecución de la tarea y el aprendizaje de todos los miembros.

4. Para que exista la colaboración es imprescindible una comunicación eficiente entre todos los miembros del grupo.

Para diseñar e implementar situaciones de aprendizaje de forma colaborativa con otros tutores, es muy importante, al menos en nuestro caso, el uso de las TIC y aprovechar el poder de la virtualidad (Domingo y Marqués, 2011). Una de las novedades que hemos utilizado para facilitar el trabajo colaborativo es el uso de los grupos de WhatsApp. El uso del WhatsApp nos facilita el intercambio rápido e instantáneo mediante texto, imágenes o videos, de lo que va sucediendo a lo largo de las situaciones. Sirve de gran ayuda, ya que nos permite realizar el seguimiento, así como compartir ideas y sugerencias de mejora sobre las tareas que se han de realizar.

\subsection{Metodología Flipped Classroom}

Una de las características del modelo de aprendizaje Flipped Classroom es invertir el proceso de aprendizaje. Un objetivo de este modelo es la individualización del 
aprendizaje, ya que mediante sus métodos dota al profesor de mucho tiempo para centrarse en la diversidad de los alumnos. Determina el uso interdisciplinar de las TICs, cuyas herramientas serán esenciales para la Flipped Classroom (Sams y Bergmann, 2013).

El mundo educativo está probando continuamente la efectividad de los nuevos recursos, y se ha comprobado ampliamente que estos métodos han resultado tanto o más útiles que la aproximación clásica (Bergmann, 2013). En base a todos estos aspectos, se decide realizar este trabajo para conocer la influencia de Flipped Classroom y su aplicación para la realización del TFG, pues consideramos que puede ser muy beneficiosa ya que facilita más tiempo para que alumnos puedan aprender los conceptos y seguir aprendiendo mediante la investigación. Autores como Bergmann y Sams (2014) y Ahmadi et al. (2011), afirman que aplicar el modelo Flipped Classroom es beneficioso para los alumnos, ya que han tenido más tiempo para llevar a cabo más actividades basadas en la investigación y para desarrollar más las implicaciones en sus proyectos.

\section{Propuesta de un modelo conceptual para la realización del TFG de forma interdisciplinar}

Aunque el rendimiento académico puede ser abordado desde distintas perspectivas, la más importante es la efectividad y corresponde al nivel de éxito en el cumplimiento de los objetivos establecidos en los programas de estudio de la asignatura. En nuestro caso utilizamos el indicador como el número de créditos aprobados respecto al número de créditos matriculados en dicha asignatura, así como la nota media obtenida por los estudiantes de las distintas líneas ofertadas para la realización del TFG (Duart et al., 2008).

Diversos estudios relacionados con el uso de las TICs determinan que se puede incidir positivamente en el rendimiento académico de los estudiantes (Raines, 2012). También hay estudios que dicen que, aunque el uso de las TICs no garantiza un rendimiento académico determinado, puede conducir al estudiante a desarrollar buenas prácticas que aporten a la consecución de logros académicos (Torres-Díaz et al., 2016). En consecuencia, el uso de las TICs constituye un factor clave que marca un antes y un después en la labor tutorial del TFG ya que posibilita una mejor comunicación entre los propios tutores, así como con los estudiantes tutelados y de esta forma contribuye de manera significativa a generar profundos cambios a la hora de desarrollar dicha asignatura. Además, el uso de las TICs otorga una nueva dimensión a la tutoría por cuanto confiere a la misma la posibilidad de adaptarse a las necesidades que se presentan en cada momento (Ferro et al., 2009). También favorece la interactividad, tanto entre los participantes en el proceso (profesor tutor y estudiantes) como en los contenidos, facilitando la enseñanza y aprendizaje de los estudiantes al generar una mejor comunicación con sus tutores (Pantoja y Zwierewicz, 2008). Esto permite que los estudiantes vayan a su propio ritmo de aprendizaje. 
En suma, podemos establecer que cuando el tutor se ciñe a un contexto de trabajo específico, como puede ser la tutorización del TFG, el uso de las TICs facilita procesos tutoriales innovadores controlando el riesgo de plagio por parte del alumnado (Heberling, 2002; Zapata, 2010). En nuestra experiencia otro método de comunicación utilizado en la tutorización ha sido la creación de un grupo de Whatsapp a través del cual los estudiantes intentan resolver de forma conjunta las dudas planteadas en la elaboración de sus trabajos y en caso de no tener claro la solución del problema, se trasladan éstas a los tutores (Türel y Toraman, 2015). Así, los estudiantes se apoyan para trabajar de forma eficiente y resolver problemas comunes de manera constructiva, ayudándose en el proceso de aprendizaje (Crossman, 2007). Esto ha favorecido que de las TICs utilizadas en la tutorización del TFG fue precisamente el grupo de Whatsapp la más empleada por los estudiantes, en segundo lugar, el UMUbox, en tercer lugar, el correo electrónico y por último el teléfono móvil. El uso de todas está herramientas ha favorecido que la metodología flipped classroom funcionara exitosamente, pues ayudó a los estudiantes a realizar determinados procesos de aprendizaje de manera autónoma con el apoyo de los materiales ofrecidos con anterioridad. De esta forma, en las sesiones de tutoría grupales se potencian otros procesos de aprendizaje y se resolvían las dudas que se planteaban. Consideramos que este método de trabajo para la elaboración del TFG es mayor el compromiso y la implicación de los estudiantes en todo el proceso de aprendizaje, pues hay una mayor retroalimentación entre ellos y los tutores. Además, lo que antes se hacía en el aula se puede hacer en cualquier otro lugar, de modo que el contacto cara a cara se hace mayor hincapié en el aprendizaje. A todas estas consideraciones, añadimos su capacidad para facilitar el aprendizaje colaborativo, ya que favorece que los estudiantes puedan crear de manera compartida un grupo de trabajo para abordar proyectos como son los del TFG.

Una vez establecidas las relaciones entre las variables propuestas en nuestro estudio sobre la realización interdisciplinar del TFG apoyado en el uso de las TICs, presentamos en la Figura 1 el modelo conceptual que las representan. 


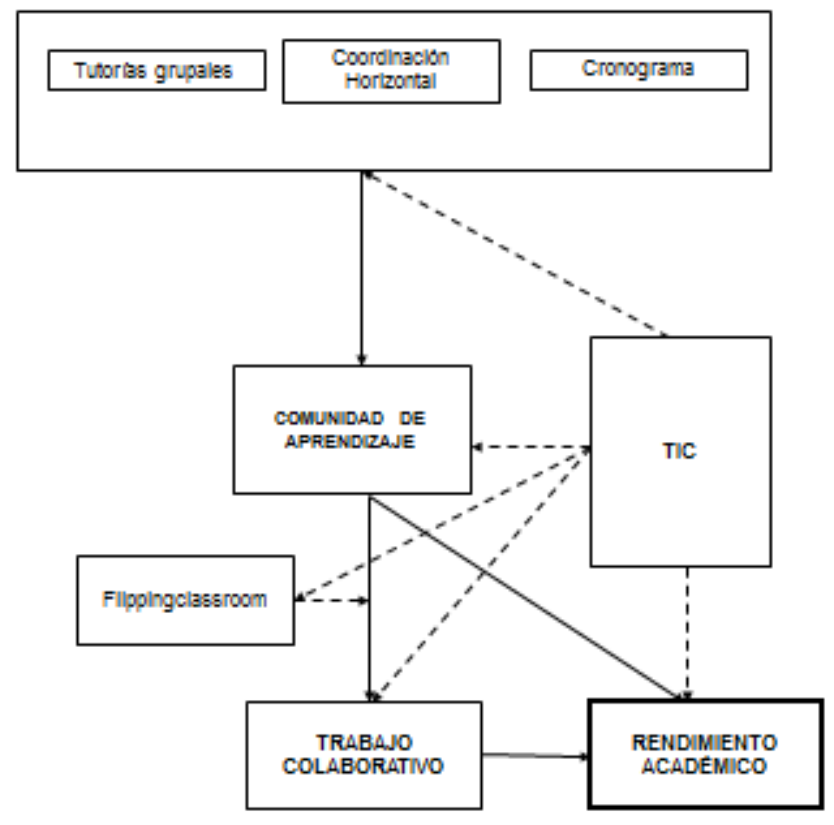

Figura 1. Modelo de innovación educativa en la realización del TFG

Podemos observar en el modelo propuesto que el uso de las TICs logra apoyar y consolidar la función de los tutores en un proceso de innovación interdisciplinar en la realización del TFG, superando las dificultades de coordinación y organización que anteriormente se planteaban entre los tutores y los estudiantes para realizar de forma eficiente dicha tarea.

\section{Objetivos del proceso de innovación docente aplicado al TFG}

El objetivo principal de este proceso de innovación docente aplicado a la gestión, seguimiento y defensa del TFG de los alumnos de la facultad de Economía y Empresa de la Región de Murcia es mejorar el rendimiento académico al incorporar las TICs como elemento clave para la comunicación entre tutores, tutores y estudiantes y entre los propios estudiantes.

También nos proponemos alcanzar los siguientes objetivos específicos:

- Orientar la enseñanza de la signatura del TFG hacia la actividad profesional.

- Favorecer una visión interdisciplinar de la realidad profesional al alumnado.

- Favorecer el autoaprendizaje de los estudiantes.

- Posibilitar el trabajo colaborativo en el alumnado, desarrollando las competencias necesarias para su futuro desarrollo profesional.

La innovación metodológica se ha realizado sobre una línea transversal de TFG denominada "Iniciativas y proyectos comerciales" a través de la coordinación de dos áreas de conocimiento (Marketing y Métodos Cuantitativos) y cotutelada por un profesor adscrito a cada una de ellas. 
Para alcanzar los objetivos propuestos en la primera etapa los estudiantes efectúan la viabilidad de un proyecto empresarial. A continuación, una vez validada la idea inicial para la constitución o relanzamiento de una empresa, los estudiantes deben revisar el trabajo realizado y elaborar una propuesta completa, incluyendo los aspectos de diseño de empresa (áreas funcionales) y de implantación, que quedará plasmada en un Plan de Empresa. Esta es la segunda y definitiva etapa que culmina con la redacción y entrega del informe de la memoria escrita del TFG y, posteriormente, la presentación y defensa del mismo.

\section{Metodología}

La línea transversal comenzó en el curso académico 2012-13, participando, en cada uno de los años en el que se está llevando a cabo esta innovación educativa, 12 estudiantes. De ellos, 6 corresponden al Grado en Administración y Dirección de Empresas y otros 6 al Grado en Marketing.

Los docentes encargados de esta nueva línea transversal establecen un cronograma de actuaciones conocido por todos los alumnos matriculados en el TFG que han optado por esta línea, en el que se especifican las tutorías grupales que se van a llevar a cabo desde febrero hasta junio, ya que el TFG es una asignatura de segundo cuatrimestre. La duración de cada tutoría grupal es de 2 horas, estableciéndose cada 15 días con un cronograma presentado a los alumnos al inicio del cuatrimestre. Se realizan un total de 8 sesiones grupales cada curso académico. En la sesión inicial, los tutores proporcionaron ideas de cómo realizar un trabajo de investigación y la elección del tema específico en la creación de proyecto empresarial, título, breve revisión bibliográfica y propósito del estudio. En la sesión 2, se realiza la elección del tema de investigación entre dos o tres alternativas. Se explica la estructura de un TFG por parte de los tutores. En la sesión 3, se realiza una exposición grupal por cada uno de los estudiantes, en la que muestran al resto del grupo el título, tipo de estudio, objetivos, variables, muestra, herramienta de recogida de datos y palabras clave de su TFG. El tutor enseña estrategias de búsquedas bibliográficas y presentación de TFG de años anteriores como ejemplos a seguir. En la sesión 4, se hace la presentación del índice, marco teórico, muestra, hipótesis o preguntas de investigación de cada estudiante. El tutor presenta el uso de normas APA y la elaboración del TFG según la guía docente de la Facultad de Economía y Empresa. En la sesión 5, los estudiantes presentan las encuestas realizadas ad-hoc como herramienta de recogida de datos y se hace una validación informal grupal. El tutor expone el uso de los programas SPSS, cómo redactar resultados, discusión y conclusiones, ofreciendo bibliografía de apoyo. En la sexta se plantean las dudas y últimos problemas surgidos en el segundo borrador por parte de cada uno de los estudiantes. En la sesión 7, presentación del tercer borrador y aclaración de las dudas sobre la defensa. En la sesión 8 y última grupal, exposición del power-point de cada TFG por cada uno de los estudiantes y recomendaciones finales.

Durante las sesiones de tutoría grupales se emplea la metodología flipped classroom mediante la cual los alumnos realizan determinados procesos de aprendizaje de manera autónoma con el apoyo de los materiales ofrecidos con anterioridad y a través del contacto más directo con el resto de compañeros. Además, es preciso mencionar que al 
alumnado se le facilitó feedback de los 3 borradores entregados durante el curso por parte de cada uno de ellos. Los tutores hicieron devoluciones corregidas de los mismos a través de sugerencias y comentarios. Este feedback que se facilitó, durante el proceso de elaboración de cada proyecto, trato de motivar a los estudiantes, para que mejorasen la calidad de sus borradores hasta llegar al documento final.

Con esta metodología se fue creando una comunidad de aprendizaje durante el desarrollo de la asignatura. La participación de los estudiantes se mantuvo constante a través del tiempo, mostrando en todo momento entusiasmo, confianza y responsabilidad en el desarrollo de las interacciones para la resolución de sus proyectos. Asimismo, los tutores guiaron en todo momento el proceso de aprendizaje a través del cronograma, la planificación del trabajo y especialmente promoviendo que todos los participantes se conocieran y pudieran colaborar entre ellos.

\section{Resultados}

Para analizar los resultados obtenidos con esta innovación metodológica se ha recopilado información de tres cursos académicos y se ha realizado un estudio comparativo con el resto de trabajos de las distintas líneas ofertadas en la Facultad de Economía y Empresa que recibieron una calificación mayor o igual a 5 puntos sobre 10. En la línea transversal el $100 \%$ de los trabajos defendidos aprobaron mientras que el resto de las líneas el porcentaje ronda el $85 \%$.

El $40 \%$ culminó la evaluación con una nota media superior 9 en la línea transversal (frente al 10,7\% del resto de líneas) y un $20 \%$ superó la evaluación con matrícula de honor (tabla 1). Estos logros estuvieron relacionados con el aprendizaje realizado a lo largo del curso, además de la disposición y actitud de los estudiantes como protagonistas.

\begin{tabular}{|l|c|c|c|c|c|c|}
\hline LínealNota & De 5 a 5,9 & De 6 a 6,9 & De 7 a 7,9 & De 8 a 8,9 & De 9 a 10 & Total \\
\hline Transversal & 0,0 & 16,7 & 20 & 23,3 & 40 & 100 \\
\hline Resto líneas & 10,0 & 22,1 & 29,4 & 27,9 & 10,7 & 100 \\
\hline
\end{tabular}

Tabla 1: Distribución porcentual de las notas del TFG según Línea

Del total de estudiantes que eligieron la línea transversal el 43,3\% es del sexo masculino y el 56,7\% restante femenino, con edades distribuidas en la franja de los 21 a 24 años. Esta distribución difiere un poco en el resto de líneas ofertadas, 48,2\% de hombres y el $51,8 \%$ son mujeres (tabla 2).

\begin{tabular}{|l|c|c|}
\hline SexolLínea & Resto & Transversal \\
\hline Hombre & $48,2 \%$ & $43,3 \%$ \\
\hline Mujer & $51,8 \%$ & $56,7 \%$ \\
\hline
\end{tabular}

Tabla 2: Porcentaje de TFGs según sexo y línea 
En el gráfico 1 se puede observar la nota media del TFG según línea y sexo, apreciándose diferencias entre los resultados de estas variables. En la línea transversal, los resultados son mejores tanto para los hombres como para las mujeres.

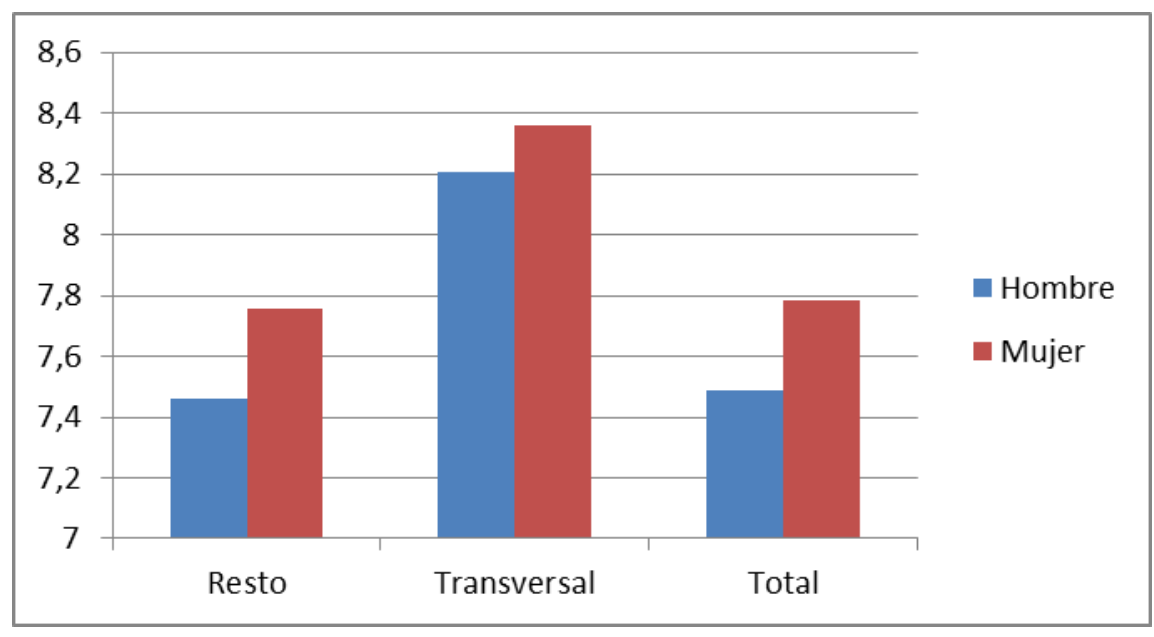

Gráfico 1: Nota media de los TFGs

Para determinar si el rendimiento en el TFG depende del sexo, la línea elegida y si es importante la interacción entre ambas variables, realizamos un ANOVA que nos permite determinar en primer lugar que no existe interacción entre sexo y línea (ver gráfico 2).

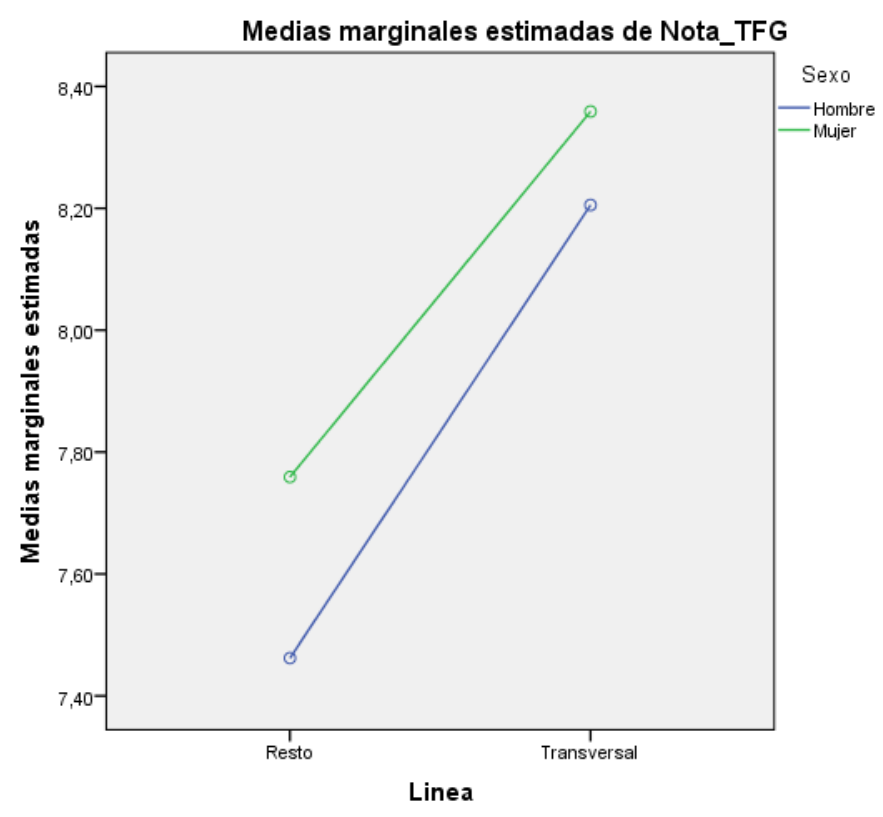

Gráfico 2: Nota media marginal estimada del TFG según línea y sexo

Si no se tiene en cuenta la interacción, encontramos diferencias significativas en el rendimiento tanto por línea como por sexo (tabla 3). Teniendo en cuenta los valores medios, la mujer obtiene mejores resultados que el hombre y en la línea transversal los resultados son superiores. 


\begin{tabular}{|l|c|c|c|c|c|}
\hline Origen & $\begin{array}{c}\text { Suma de cuadrados tipo } \\
\text { III }\end{array}$ & gl & $\begin{array}{c}\text { Media } \\
\text { cuadrática }\end{array}$ & F & Sig. \\
\hline $\begin{array}{l}\text { Modelo } \\
\text { corregido }\end{array}$ & $28,889^{\mathrm{a}}$ & 2 & 14,445 & 11,720 & 0,000 \\
\hline Intersección & 7254,428 & 1 & 7254,428 & 5886,055 & 0,000 \\
\hline Sexo & 15,718 & 1 & 15,718 & 12,753 & 0,000 \\
\hline Línea & 12,623 & 1 & 12,623 & 10,242 & 0,001 \\
\hline Error & 910,801 & 739 & 1,232 & & \\
\hline Total & 44286,949 & 742 & & & \\
\hline Total corregida & 939,690 & 741 & & & \\
\hline
\end{tabular}

Tabla 3: Anova sobre la nota del TFG con los factores sexo y línea

Si realizamos el estudio para cada línea por separado (tabla 4), se observa cómo el sexo discrimina en el conjunto de resto de líneas ( $p$-valor=0,000), mientras que no ocurre lo mismo con la transversal $(\mathrm{p}=0,713)$.

\begin{tabular}{|c|c|c|c|c|c|c|}
\hline Línea & Origen & $\begin{array}{c}\text { Suma de } \\
\text { cuadrados tipo III }\end{array}$ & Gl & $\begin{array}{c}\text { Media } \\
\text { cuadrática }\end{array}$ & $\mathrm{F}$ & Sig. \\
\hline \multirow{6}{*}{ Resto } & $\begin{array}{l}\text { Modelo } \\
\text { corregido }\end{array}$ & $15,691^{\mathrm{a}}$ & 1 & 15,691 & 12,723 & 0,000 \\
\hline & Intersección & 41183,747 & 1 & 41183,747 & 33394,736 & 0,000 \\
\hline & Sexo & 15,691 & 1 & 15,691 & 12,723 & 0,000 \\
\hline & Error & 875,601 & 710 & 1,233 & & \\
\hline & Total & 42188,838 & 712 & & & \\
\hline & Total corregida & 891,292 & 711 & & & \\
\hline \multirow{6}{*}{ Transversal } & $\begin{array}{l}\text { Modelo } \\
\text { corregido }\end{array}$ & $0,173^{\mathrm{b}}$ & 1 & 0,173 & 0,139 & 0,713 \\
\hline & Intersección & 2021,214 & 1 & 2021,214 & 1614,494 & 0,000 \\
\hline & Sexo & 0,173 & 1 & 0,173 & 0,139 & 0,713 \\
\hline & Error & 35,054 & 28 & 1,252 & & \\
\hline & Total & 2098,111 & 30 & & & \\
\hline & Total corregida & 35,227 & 29 & & & \\
\hline
\end{tabular}

Tabla 4: Anova sobre la nota del TFG para el factor sexo, en cada una de las líneas

Por tanto, podemos afirmar que los estudiantes de la línea transversal terminaron el TFG de manera muy positiva, reconociendo los pilares básicos en los que se había fundamentado las tutorías. El primero basado en el trabajo colaborativo entre ellos fomentado desde las tutorías grupales y el segundo la buena planificación apoyada en la comunicación y en el uso de las TICs, que le ha permitido superar con más facilidad las situaciones que le iban surgiendo en la elaboración de su proyecto. Además, esta metodología empleada para la elaboración del TFG no discrimina por sexo. Es decir, según distintas investigaciones las mujeres obtienen mejores calificaciones en todos los 
grados, desde la educación infantil a la etapa universitaria, Así lo demuestra por ejemplo el estudio realizado entre 1914 y 2011 con datos de un millón de estudiantes en más de 30 países, llevado a cabo por los psicólogos Daniel y Susan Voyer, de la Universidad de New Bronswich. Donde se afirma en sus conclusiones que el rendimiento académico de las mujeres es superior. Estos resultados que destacan el mejor rendimiento académico de las mujeres prácticamente en todos los países del mundo, plantea una pregunta: ¿por qué las mujeres obtienen mejores notas? Al parecer una de las diferencias fundamentales es que las mujeres aún con la misma edad tienen un mayor grado de 'madurez', lo que les facilita la posibilidad de planificarse mejor, establecen metas académicas muy claras y, además, están dispuestas a sacrificarse y esforzarse para lograrlas. Según los resultados de nuestro estudio podemos decir que estas competencias más afianzadas en las alumnas influyen en sus compañeros varones, los cuales trabajan el desarrollo de su proyecto del TFG al mismo nivel de esfuerzo y dedicación que sus compañeras. Esforzándose y comprometiéndose en los plazos de avance del proyecto, hasta la finalización del mismo. Por ello no hemos encontrado diferencias estadísticamente significativas entre hombres y mujeres que han utilizado nuestra metodología, no así en el resto de las líneas ofertadas del TFG, donde las mujeres han obtenido mejores calificaciones que sus compañeros varones.

\section{Discusión y conclusiones}

En este trabajo hemos analizado un modelo conceptual de dimensiones teóricas capaz de revelar los procesos que influyen en un mejor rendimiento académico de los estudiantes en la realización del TFG. Del análisis de los resultados obtenidos podemos extraer las siguientes conclusiones:

1) El uso de las TICs incide en la mejora del dinamismo de los procesos de enseñanza aprendizaje en el TFG pues favorecen la conectividad de estudiantes $\mathrm{y}$ tutores en cualquier momento y en cualquier lugar, tanto dentro de los contextos de aprendizaje formal como en los no formales. Además, la aplicación de WhatsApp genera nuevos enfoques en las concepciones pedagógicas, pues bien utilizado académicamente, fomenta la investigación y el autoaprendizaje en el estudiante.

2) La inclusión de las TICs como medio de comunicación ha logrado consolidar la función de la tutoría. Pues a los estudiantes el uso de las TICs les facilita mantenerse en contacto directo y mantener una comunicación con sus tutores y compañeros. Podemos decir que del uso de las TICs puede ser el complemento ideal para la acción tutorial. Ya que les facilita a los estudiantes desarrollar habilidades de comunicación en equipo, permitiéndoles una diversidad de enfoques muy enriquecedora para llevar a cabo la elaboración del TFG.

Nuestro trabajo tiene claras implicaciones académicas y prácticas ya que se facilita la coordinación interdisciplinar permitiendo un aprendizaje integrador mediante la realización de actividades que recogen aspectos de diferentes asignaturas de la titulación. Por otra parte, se fomenta la coordinación y optimización del trabajo realizado por el alumno. Además, se desarrollan metodologías activas de aprendizaje. Considerando estas implicaciones de nuestro estudio y atendiendo la problemática que 
se vive hoy en día en la acción tutorial de los TFGs, podemos establecer las siguientes propuestas:

a) Con respecto al profesorado, la coordinación de éste en cada curso académico en torno a una temática, ha supuesto la puesta en común entre el profesorado de los conocimientos impartidos y la conciencia de la necesidad de complementación para alcanzar un mismo objetivo, por tanto, la coordinación se ha extendido más allá de la experiencia concreta de modo que el profesorado ha sido consciente de la necesidad de trabajo en equipo y la coordinación para un correcto desarrollo del TFG.

b) Con respecto al alumnado, con esta experiencia, se ha aproximado a su futura práctica profesional, en cuanto que ha trabajado de forma interdisciplinar. Además, el alumnado ha percibido en sus tutores conexión y coordinación en los contenidos de diversas materias.

c) Se debería fomentar el uso del Whatsapp para la acción tutorial. Para que de esta manera los estudiantes puedan seguir las recomendaciones y sugerencias de su tutor al estar en contacto directo en tiempo real con él. Las múltiples ventajas que plantea la utilización de WhatsApp en entornos de enseñanza- aprendizaje nos permiten hablar de esta herramienta como un interesante instrumento para diseñar un modelo de educación basado en la construcción colectiva del aprendizaje y la colaboración.

A pesar de las implicaciones anteriores, nuestro trabajo no está exento de limitaciones. La principal limitación es que nuestra experiencia de innovación educativa se puede considerar "piloto", a pesar de haberse realizado durante 3 cursos académicos consecutivos. No obstante, en un futuro se pretende replicar la misma investigación para grupos más amplios de alumnos, contando así con un mayor número de estudiantes participantes.

A modo de resumen podemos decir que es importante que el uso de las TICs en la Educación Superior vaya acompañado de cambios en la concepción pedagógica por parte de los tutores. Para ello, han de asumir el reto de incorporar desde metodologías innovadoras hasta nuevos recursos tecnológicos en el proceso de enseñanza-aprendizaje. Ya que se puede afirmar, según los resultados de nuestro estudio, que el uso de TICs favorece las propuestas de innovación educativa que requieren una mayor coordinación entre distintos colectivos a través de una comunicación más efectiva, como es el que caso de esta propuesta de innovación en la elaboración del TFG, ya que se consigue promover el autoaprendizaje, la interacción y la solidaridad entre los estudiantes, obteniendo unos resultados de éxito en sus calificaciones finales, mayor igualdad en las notas y que no halla diferencia de sexo en los resultados académicos alcanzado por los estudiantes. Por eso no es de extrañar que, al término de esta experiencia de innovación educativa, uno de los aspectos más valorados por los estudiantes haya sido la integración de conocimientos y el trabajo en equipo. Sin duda alguna, esto supone que se ha favorecido la adquisición de competencias que van a ser claves para el desarrollo de su futuro profesional. 
Presentación del artículo: 15 de febrero de 2017 Fecha de aprobación: 14 de marzo de 2017 Fecha de publicación: 31 de marzo de 2017

Faura-Martínez, U., Martín-Castejón, P.J. y Lafuente-Lechuga, M. (2017). Un modelo conceptual para la realización del Trabajo Fin de Grado apoyado en el uso de las TICs. RED. Revista de Educación a Distancia, 53. Consultado el (dd/mm/aaaa) en http://www.um.es/ead/red/

Esta investigación no ha recibido ninguna subvención específica de los organismos de financiación en los sectores públicos, comerciales o sin fines de lucro.

\section{Referencias}

Ahmadi, S., Keshavarzi, A., y Foroutan, M. (2011). The application of information and communication technologies (ICT) and its relationship with improvement in teaching and learning. Procedia-Social and Behavioral Sciences, 28, 475-480.

Arbizu, F., Lobato, C., y del Castillo, L. (2005). Algunos modelos de abordaje de la tutoría universitaria. Revista de Psicodidáctica, 10(1), 7-22.

Arteaga, B., Ahedo, J., Gutiérrez, S. y Solera, E. (2013). El trabajo fin de grado maestro: modalidad online con evaluación presencial. RED. Revista de Educación a Distancia, 38. Consultado el [06/03/2017] en http://www.um.es/ead/red/38

Artola, C (2012) Estrategias para el trabajo colaborativo en el salón de clase con el uso de la tecnología. Slideshare. Recuperado de https://es.slideshare.net/ceciartola/trabajo-colaborativo-slideshare-13090271

Barquilla, F (2016) Estudio sobre el estado de internet y las redes sociales en 2016. El Tipómetro. Recuperado de http://franbarquilla.com/estudio-sobre-el-estado-deinternet-y-las-redes-sociales-en-2016/

Bergmann, J. (2013). The Perfect Match: Flipped Learning \& the Common Core. Pearson \& Innovation Network.

Bergmann, J., y Sams, A. (2014) Flipping For Mastery. Educational Leadership, 71(4), 24-29.

Bonilla, M.J., Fuentes, L., Vacas, C., y Vacas, T. (2012). Análisis del proceso de evaluación del Trabajo Fin de Grado en las nuevas titulaciones. Revista de Educación en Contabilidad, Finanzas y Administración de Empresas, 3, 5-12.

Bote, M., Lafuente-Lechuga M., y Puigcerver M.C. (2013). Manual del TFG en la Facultad de Economía y Empresa. Curso 2013-2014. DIGITUM. Recuperado de http://www.um.es/documents/16162/1727061/manual-TFG+13-14.pdf/a03737ee3fc8-40a9-a808-c2e0c7c6acb6

Castaño, J. (2011). El uso de Internet para la interacción en el aprendizaje: Un análisis de la eficacia y la igualdad en el sistema universitario catalán. Barcelona: 
Universitat Oberta de Catalunya. Recuperado de http://www.tesisenred.net/bitstream/handle/10803/52561/Tesis_Jonatan_Casta\%C3 $\%$ B1o.pdf?sequence $=1$

Cenich, G., y Santos, G. (2005). Propuesta de aprendizaje basado en proyecto y trabajo colaborativo: experiencia de un curso en línea. Revista Electrónica de Investigación Educativa. Recuperado de: http://redie.uabc.mx/redie/article/viewFile/133/228

Coll, C. (2001). Las Comunidades de Aprendizaje y el futuro de la educación: el punto de vista del Fórum Universal de las Culturas Barcelona-2004. Simposium Internacional sobre las Comunidades de Aprendizaje.

Crossman, J. (2007). The role of relationships and emotions in student perceptions of learning and assessment. Higher Education Research and Development, 26(3), 31327.

Domingo, M. y Marquès, P. (2011). Aulas 2.0 y uso de las TIC en la práctica docente. Comunicar, 37, 169-175.

Duart, J. M., Gil, M., Puyol, M., \& Castaño, J. (2008). La universidad en la sociedad Red. Barcelona: Ariel.

Ferro, C.A., Martínez, A.I., y Otero, M.C. (2009). Ventajas del uso de las TICs en el proceso de enseñanza-aprendizaje desde la óptica de los docentes universitarios españoles. Revista Electrónica de Tecnología Educativa, EDUTEC, 29.

García-Nieto, N. (2008). La función tutorial de la Universidad en el actual contexto de la Educación Superior. Revista interuniversitaria de formación del profesorado, 61, 21-48.

Garzozi, R., y Lucas, J. (2014). Experiencia de implementación de entornos virtuales de aprendizaje como estrategia para la culminación del estudio de posgrado Auditoria Integral en la Universidad Técnica Particular de Loja, Ecuador. Tecnología Educativa, Revista CONAIC, 1, 70-79. Recuperado de http://conaic.net/revista/publicaciones/Articulo5_Vol1_Num1.pdf

Heberling, M. (2002). Maintaining academic integrity in online education. Online Journal of Distance Learning Administration, 5(2). Recuperado de http://www.westga.edu/ distance/ojdla/spring2002/heberling51.htm

Lara, J.A., Lizcano, D., Martínez, M.A., Pazos, J., y Riera, T. (2014). A system for knowledge discovery in e-learning environments within the European Higher Education Area. Application to student data from Open University of Madrid, UDIMA. Computers \& Education, 72, 23-36.

Mishra, S., Draus, P., Goreva, N., Leone, G., y Caputo, D. (2014). The Impact of Internet Addiction on University Students and Its Effect on Subsequent Academic Success: A Survey Based Study. Issues in Information Systems, 15(I), 344-352. Recuperado de http://iacis.org/iis/2014/67_iis_2014_344-352.pdf.

Obaya, V. A., y Vargas, R. Y. M. (2014). La tutoría en la educación superior. Educación Química, 25(4), 478-487. 
Olaskoaga-Larrauri, J., González-Laskibar, X., Marúm-Espinosa, E., y OnaindiaGerrikabeitia, E. (2015). Reformas organizativas en las instituciones de educación superior, condiciones laborales y reacciones de los académicos. Revista Iberoamericana de Educación Superior (RIES), VI (17), 102-118.

Onrubia, J. (2004). Las aulas como comunidades de aprendizaje. Revista Electrónica Trabajadores de la Enseñanza T.E. Federación de Enseñanza CC.OO, 249, 14-15. Recuperado de https://www.innova.uned.es/webpages/educalia/Comunidades\%2520de\%2520apren dizaje\%2520pag\%252012\%2520al\%252017.pdf

Pantoja, A., y Zwierewicz, M. (2008). Procesos de orientación en entornos virtuales de aprendizaje. Revista Española de Orientación y Psicopedagogía, 19 (3), 282-290. Recuperado de http://revistas.uned.es/index.php/reop/article/view/11429/10927

Pantoja, A., y Villanueva. C. (2010). Recursos tecnológicos aplicados a la tutoría. En M. Álvarez y M. Bisquerra (Coords.), Manual de Orientación y Tutoría. CD nº 40. Barcelona: Wolters Kluwer.

Pedraza, N., Farías, G., Lavín, J., y Torres, A. (2013). Las competencias docentes en TIC en las áreas de negocios y contaduría. Perfiles Educativos, vol. XXXV, 139, IISUE-UNAM. Recuperado de http://www.scielo.org.mx/pdf/peredu/v35n139/v35n139a2.pdf

Puigcerver, M.C.; Martín, P. J., y Antón M. (2013). Los beneficios de la coordinación horizontal en la realización de los TFG, para los estudiantes de Grado en Administración y Dirección de Empresas. Revista de Investigación en Educación $11(1), 97-117$.

Raines, J. (2012). The Effect of Online Homework Due Dates on College Student Achievement in Elementary Algebra. Journal of Studies in Education, 2(3), 1-18.

Rekalde, I. (2011). ¿Cómo afrontar el trabajo fin de grado? Un problema o una oportunidad para culminar con el desarrollo de las competencias. Revista Complutense de Educación, 22, 179-193.

Ricoy, M.C. y Fernández, J. (2013). Contribuciones y controversias que genera el uso de las TIC en la Educación Superior: un estudio de caso. Revista de Educación, 360, 509-532. Recuperado de http://www.revistaeducacion.mec.es/doi/360_125.pdf

Rodríguez, M.A., y Llanes, L. (coords.) (2013). Cómo elaborar, tutorizar y evaluar un Trabajo Fin de Máster, Agència per a la Qualitat del Sistema Universitari de Catalunya. Recuperado de http://www.aqu.cat/doc/doc_18533565_1.pdf

Rullán, M., Fernández, M., Estapé, G. y Márquez, M.D. (2010). La evaluación de competencias transversales en la materia Trabajos Fin de Grado. Un estudio preliminar sobre la necesidad y oportunidad de establecer medios e instrumentos por ramas de conocimiento. Revista de Docencia Universitaria, 8(1), 74-100.

Sams, A., y Bergmann, J. (2013). Flip Your Students' Learning. Educational Leadership, 70(6), 16-20. 
Torres-Díaz J.C., Duart J.M., Gómez-Alvarado H.F., Marín-Gutiérrez I., y SegarraFaggioni V. (2016). Usos de Internet y éxito académico en estudiantes universitarios. Comunicar, 48, 61-70.

Türel, Y.K., y Toraman, M. (2015). The Relationship between Internet Addiction and Academic Success of Secondary School Students. Antropologist, 20, 280-288. Recuperado de http://krepublishers.com/02-Journals/T-Anth/Anth-20-0-000-15Web/Anth-20-1-000-15-Abst-PDF/T-ANTH-20-1,2-280-15-1442-Turel-Y-K/TANTH-20-1,2-280-15-1442-Turel-Y-K-Tx[30].pdf

Wisker, G. (coord.) (2012). Trabajar individualmente con cada estudiante. Tutoría personalizada, coaching, mentoría y supervisión en Educación Superior. Madrid: Narcea.

Zapata, M. (2010). Estrategias de evaluación de competencias en entornos virtuales de aprendizaje. RED. Revista de Educación a Distancia. Sección de Docencia Universitaria en la Sociedad del Conocimiento. 1. Recuperado de https://www.um.es/ead/reddusc/1/eval_compet.pdf 